\title{
Rare variant analysis of blood pressure phenotypes in the Genetic Analysis Workshop 18 whole genome sequencing data using sequence kernel association test
}

\author{
Cates Mallaney ${ }^{\dagger}$, Yun Ju Sung ${ }^{* \dagger}$ \\ From Genetic Analysis Workshop 18 \\ Stevenson, WA, USA. 13-17 October 2012
}

\begin{abstract}
Sequence kernel association test (SKAT) has become one of the most commonly used nonburden tests for analyzing rare variants. Performance of burden tests depends on the weighting of rare and common variants when collapsing them in a genomic region. Using the systolic and diastolic blood pressure phenotypes of 142 unrelated individuals in the Genetic Analysis Workshop 18 data, we investigated whether performance of SKAT also depends on the weighting scheme. We analyzed the entire sequencing data for all 200 replications using 3 weighting schemes: equal weighting, Madsen-Browning weighting, and SKAT default linear weighting. We considered two options: all single-nucleotide polymorphisms (SNPs) and only low-frequency SNPs. A SKAT default weighting scheme (which heavily downweights common variants) performed better for the genes in which causal SNPs are mostly rare. This SKAT default weighting scheme behaved similarly to other weighting schemes after eliminating all common SNPs. In contrast, the equal weighting scheme performed the best for MAP4 and FLT3, both of which included a common variant with a large effect. However, SKAT with all 3 weighting schemes performed poorly. Overall power across all causal genes was about 0.05 , which was almost identical to the type I error rate. This poor performance is partly due to a small sample size because of the need to analyze only unrelated individuals. Because a half of causal SNPs were not found in the annotation file based on the 1000 Genomes Project, we suspect that performance was also affected by our use of incomplete annotation information.
\end{abstract}

\section{Background}

Common variants have long been thought to have a major role in common phenotypes; however, genomewide association studies (GWAS) that focused on common variants have explained only a small proportion of heritability. Because rare variants are expected to have larger effect sizes than common variants, they may explain a proportion of the missing heritability [1]. As reviewed by Bansal et al [2], because of limited power for detecting the individual effects of rare variants, several approaches for identifying them have used

\footnotetext{
* Correspondence: yunju@wubios.wustl.edu

† Contributed equally

Division of Biostatistics, Washington University in St. Louis, School of
} Medicine, St. Louis, MO 63110, USA

collapsing methods (called burden tests) that assess the combined effect of multiple rare variants in a genomic region.

Because these burden tests implicitly assume that all variants influence the trait in the same direction and with the same magnitude of effect (after incorporating known weights), their power is reduced if certain variants confer increased risk while others are protective [3]. Several methods have been developed recently that are less sensitive to this mixture of rare-variant effects. Two one-sided statistics by Ionita-Laza et al [4] quantify enrichment in risk variants and protective variants, respectively. The C-alpha test by Neale et al [5] uses the distribution of genetic variation observed in cases and
controls to detect the presence of a mixture, thus 
implicating genes as risk factors for disease. By testing the variance rather than the mean, the $\mathrm{C}$-alpha test maintains consistent power when the target set contains both risk and protective variants [5]. The sequence kernel association test (SKAT) by Wu et al [6] is a score-based variance component test for association of variants in a region for continuous and discrete traits, which easily adjusts for covariates.

Performance of burden tests has been shown to depend on how rare and common variants are weighted when collapsing them in a genomic region [2,7]. In this article, using the Genetic Analysis Workshop 18 (GAW18) sequence data, we investigated whether performance of the most commonly used nonburden test, SKAT, also depends on the weighting scheme. In particular, we evaluated 3 weighting schemes: equal weighting, MadsenBrowning weighting, and SKAT default linear weighting. Analyses were performed without knowledge of the underlying simulation model. However, we used the GAW18 answers in presenting the results below.

\section{Methods}

\section{Sequence and phenotypic data}

GAW18 provided whole genome sequencing (WGS) data and longitudinal phenotype data for related individuals of Mexican American heritage. Because SKAT can only handle unrelated individuals, we used both sequence and phenotype data on a subset of 142 unrelated individuals. Using PLINK [8], we extracted the data and created input files for SKAT. We used the first-visit measurements for diastolic blood pressure (DBP) and systolic blood pressure (SBP) for all 200 simulated data and the most likely genotype data based on sequencing (geno.csv files). For covariates of both DBP and SBP, we used age, sex, blood pressure medication, and smoking.

\section{Annotation files}

To obtain a gene list to run SKAT, we used the annotation file that was constructed based on the 1000 Genomes Project (http://www.sph.umich.edu/csg/abecasis/MACH/ download/1000G-2010-06.html). Of the 8,348,674 singlenucleotide polymorphisms (SNPs) in the data set, $2,652,577$ were located in genes, with a total of 10,922 genes. Some of these SNPs belonged to multiple overlapping genes and were used for multiple genes in which they were contained. Because 1,856,005 SNPs in the genotype files were not found in the 1000 Genomes annotation file, not all genes were available for testing. For the SBP phenotype, we were missing 4 causal genes, and for the DBP phenotype, we were missing 3 causal genes.

\section{Statistical analysis}

We applied SKAT to the analysis of all 200 replicates of simulated DBP and SBP phenotypes for 2,652,577 SNPs that are located in genes, spanning all odd-numbered chromosomes provided by GAW18. Because our analysis was based on 142 unrelated individuals, instead of using minor allele frequency (MAF) provided by GAW18 (which is based on 959 related individuals), we computed MAF based on these 142 individuals using PLINK [8]. We also constructed a data set including low-frequency SNPs (with our computed MAF <0.05).

For a continuous trait $Y$, SKAT uses a linear model $Y_{i}=\gamma_{0}+\gamma_{1} X_{i}+\beta G_{i}$ with genotype values $G_{i}$ and covariates $X_{i}$ for subject $i$. As described by Wu et al [6], SKAT assumes that the genetic effect $\beta_{j}$ of an individual variant $j$ follows an arbitrary distribution with mean 0 and variance $w_{j} \tau$, where $\tau$ is a variance component and $w_{j}$ is a prespecified weight for variant $j$. SKAT further assumes that $\sqrt{w_{j}}$ follows $\operatorname{Beta}\left(M A F_{j} ; a_{1}, a_{2}\right)$. Weighting with $a_{1}=a_{2}=1$ corresponds to equally weighting all variants regardless of their MAF, which was shown to be equivalent to the $C$-alpha test by Neale at al [5]. Weighting with $a_{1}=a_{2}=0.5$ is the same as the weight used by Madsen and Browning [7]. Default linear weighting by SKAT uses $a_{1}=1$ and $a_{2}=25$, which puts more weight on rare variants than Madsen-Browning (M-B) weighting, as shown in Figure 1.

For both data sets (one with all SNPs and another with only low-frequency SNPs), we ran SKAT using these 3 weighting schemes: equal weighting, Madsen-Browning weighting, and SKAT default linear weighting. For each scenario, we obtained $p$-values for the 10,922 genes in 200 replications. To evaluate performance, we computed power (true-positive) and type I error (false-positive) rates

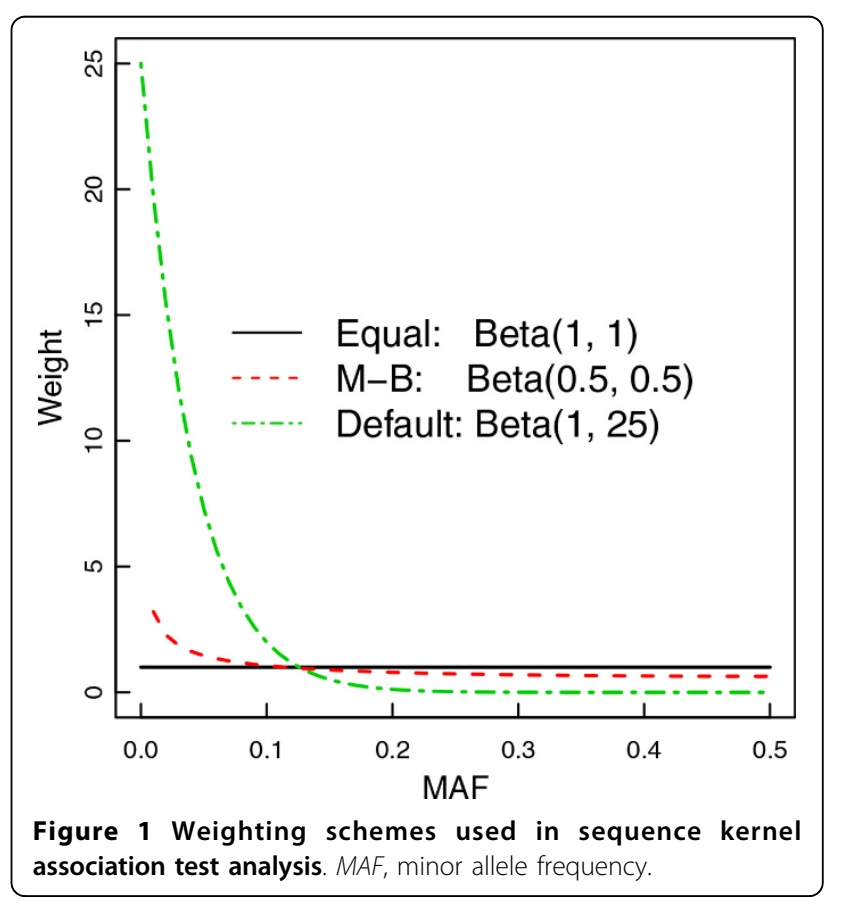


at level 0.05 . For each gene, power was computed by the proportion of replicates with $p<0.05$ over 200 replicates. The overall power was computed by averaging these values across all causal genes. Type I error was computed by averaging these values across all null genes.

\section{Results}

\section{Overall empirical power and type I error}

Table 1 presents type I error and overall power using 3 weighting schemes for both data sets. All 3 weighting schemes performed poorly. No causal gene met the threshold for genome-wide significance, and the lowest $p$-value for a true positive occurred at $1.0 \times 10^{-5}$ for analyses of SBP and $1.0 \times 10^{-4}$ for analyses of DBP. The type I error was slightly below 0.05 , roughly keeping the level 0.05 . Overall power across all causal genes was about 0.05 , which was almost identical to the type I error rate. The equal weighting scheme using all SNPs performed slightly better for both DBP and SBP phenotypes.

\section{Empirical power at causal genes}

Although overall power was similar across the 3 weighting schemes, power at each causal gene varied greatly and depended on the weighting scheme. When using all SNPs, results were considerably different across 3 weighting schemes, as shown in the left plot of Figure 2. In particular, the correlation between power using equal weighting and power using default weighting was 0.17 (shown in Table 2). In contrast, when using rare SNPs, results were more consistent across different weighting schemes, as shown in the right plot of Figure 2. Results using default weighting were very similar when using all SNPs and when using only rare SNPs (with correlation 0.97).

To understand how power depended on the weighting scheme, we selected causal genes that show power over 0.2 from at least one of weighting schemes from Figure 2; these selected causal genes are presented in Table 3. For genes TXNIP, GLUL, PDCD6IP, DGKE, and SCAP, the SKAT default weighting gave much higher power than equal weighting. Except for PDCD6IP, all causal SNPs were low frequency (with MAF $<0.05$ ). Using only low-frequency SNPs provided similar performance.

However, for genes MAP4, FLNB, DHX8, FGR, S100A6, and KRT23, the equal weighting scheme gave much higher power than the SKAT default weighting (0.32 vs. 0.04 at MAP4 for DBP). Looking within the top 20 causal variants, we found that MAP4 has 6 of the top 10 variants with the greatest effect on SBP and DBP. For all of these variants, one was common with a MAF of 0.38 , and the other 5 were low frequency (with MAF $<0.05)$. Similarly, gene FLT3 had 2 variants that fell within the top 20 causal variants, one common with a MAF of 0.42 and one rare with a MAF of 0.0016 . No other causal gene had more than one variant in the top 20 causal variants. Because of this, we suspect that equal weighting performed better than SKAT default weighting, which gives a much higher weight to rarer variants.

We also considered whether performance would depend on the signal-to-noise ratio, defined as the number of causal variants divided by the total number of variants in the gene, for each of the top 15 genes. For DBP, MAP4 had the 10th lowest sig signal-to-noise ratio, and FLT3 had the 13th lowest. For the SBP phenotype, they were 7 th and $10^{\text {th }}$, respectively. Therefore, we found that signal-to-noise ratio could not account for the relatively high performance on MAP4 and FLT3.

\section{Discussion and conclusions}

SKAT has become one of the most commonly used nonburden tests for analyzing rare variants because it is fast and because nonburden tests are shown to be more powerful when most variants are noncausal or the effects of causal variants are in different directions. Using SKAT, we were able to analyze the entire sequencing data for all 200 replications of both DBP and SBP simulated phenotypes under six different cases (3 weighting schemes times two data sets: one with all SNPs another with only lowfrequency SNPs). Performance of burden tests has been shown to depend on how to weigh rare and common variants when collapsing them in a genomic region. In this article, using the GAW 18 sequence data, we found that performance of the nonburden test SKAT also depended on the weighting scheme.

In this article, we focused on the ability of SKAT to detect genes with various MAF-based weighting schemes. When causal SNPs within a gene are mostly rare, then MAF-based weighting by upweighting rare variants and downweighting common variants would be

Table 1 Empirical power and type I error at $\alpha=0.05$ for diastolic blood pressure and systolic blood pressure phenotypes

\begin{tabular}{|c|c|c|c|c|c|c|c|c|c|c|c|c|}
\hline \multirow{3}{*}{$\begin{array}{l}\text { Phenotype } \\
\text { SNPs } \\
\text { Weight }\end{array}$} & \multicolumn{6}{|c|}{ DBP } & \multicolumn{6}{|c|}{ SBP } \\
\hline & \multicolumn{3}{|c|}{ All } & \multicolumn{3}{|c|}{ Rare } & \multicolumn{3}{|c|}{ All } & \multicolumn{3}{|c|}{ Rare } \\
\hline & Equal & M-B & Default & Equal & M-B & Default & Equal & M-B & Default & Equal & M-B & Default \\
\hline Type I Error & 0.047 & 0.043 & 0.043 & 0.044 & 0.042 & 0.043 & 0.048 & 0.048 & 0.049 & 0.048 & 0.050 & 0.049 \\
\hline Power & 0.053 & 0.050 & 0.048 & 0.048 & 0.050 & 0.048 & 0.054 & 0.053 & 0.053 & 0.049 & 0.052 & 0.052 \\
\hline
\end{tabular}

Bolded text indicates the highest power across 6 analysis options for each phenotype.

$D B P$, diastolic blood pressure; $M-B$, Madsen-Browning; $S B P$, systolic blood pressure; $S N P$, single-nucleotide polymorphism. 

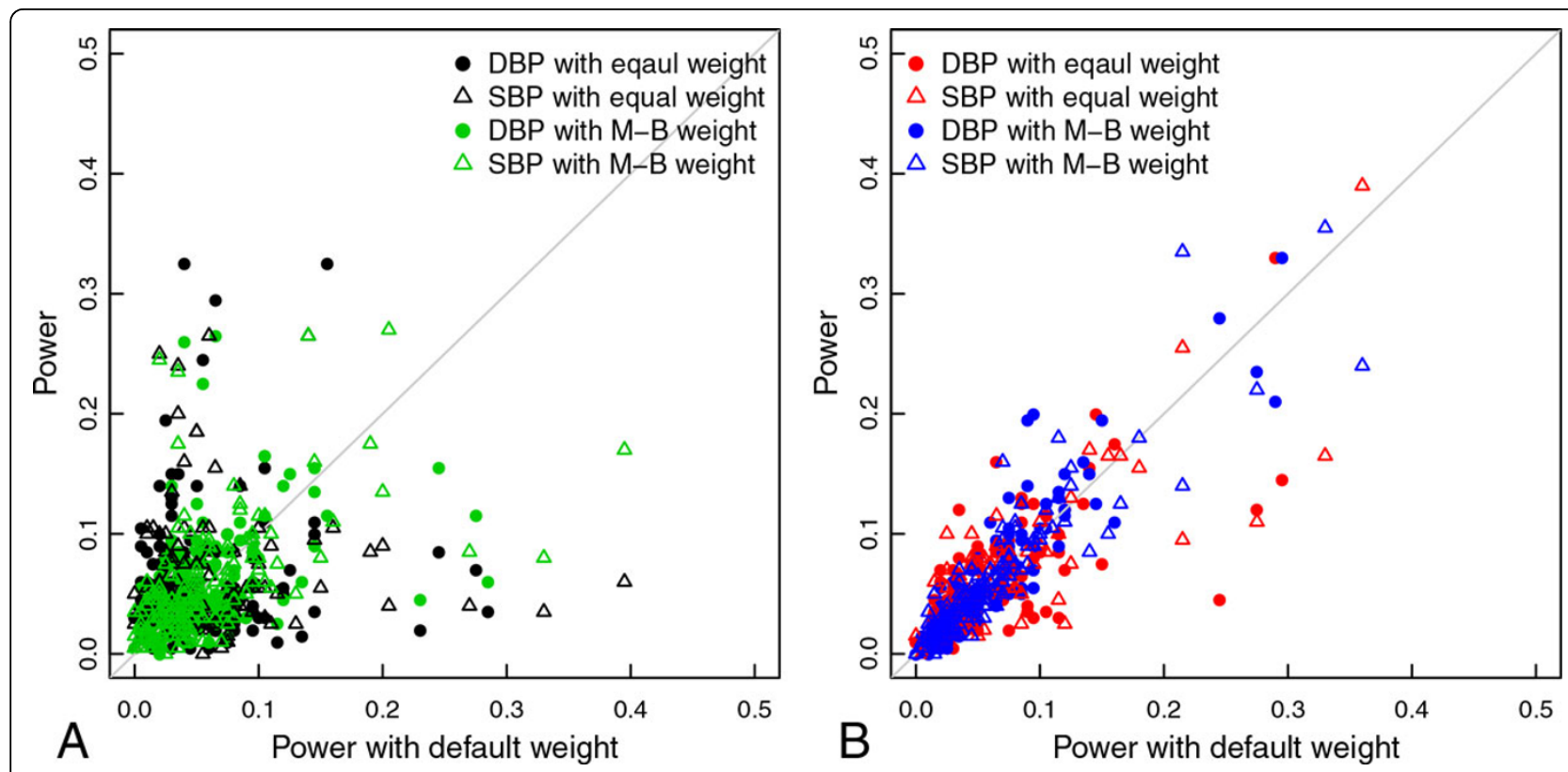

Figure 2 Power comparison using 3 weighting schemes. (A) All single-nucleotide polymorphisms (SNPs). (B) Rare SNPs. DBP, diastolic blood pressure M-B, Madsen-Browning; SBP, systolic blood pressure.

Table 2 Spearman correlation across empirical powers at all causal genes

\begin{tabular}{|c|c|c|c|c|c|c|c|}
\hline \multicolumn{2}{|c|}{ Correlation } & \multicolumn{3}{|c|}{ All } & \multicolumn{3}{|c|}{ Rare } \\
\hline & & Equal & M-B & Default & Equal & M-B & Default \\
\hline \multirow[t]{3}{*}{ All } & Equal & & 0.69 & 0.17 & 0.13 & 0.11 & 0.11 \\
\hline & $M-B$ & & & 0.61 & 0.47 & 0.55 & 0.54 \\
\hline & Default & & & & 0.79 & 0.87 & 0.95 \\
\hline \multirow[t]{3}{*}{ Rare } & Equal & & & & & 0.70 & 0.80 \\
\hline & $M-B$ & & & & & & 0.91 \\
\hline & Default & & & & & & \\
\hline
\end{tabular}

Bolded text indicates the correlation greater than 0.8 .

$M-B$, Madsen-Browning

Table 3 Causal genes with empirical power over 0.2 using any weighting scheme

\begin{tabular}{|c|c|c|c|c|c|c|c|c|c|c|c|}
\hline \multirow[t]{2}{*}{ Causal Genes } & \multicolumn{2}{|c|}{ Number of SNPs } & \multicolumn{2}{|c|}{ Number of causal SNPs ${ }^{1}$} & \multirow[t]{2}{*}{ Total \% variance explained ${ }^{2}$} & \multicolumn{3}{|c|}{ All } & \multicolumn{3}{|c|}{ Rare } \\
\hline & $\overline{\text { All }}$ & Rare & All & Rare & & Equal & M-B & Default & Equal & M-B & Default \\
\hline \multicolumn{12}{|l|}{$\overline{\mathrm{DBP}}$} \\
\hline MAP4 & 542 & 392 & 6 & 5 & 0.04826 & 0.30 & 0.27 & 0.07 & 0.09 & 0.06 & 0.07 \\
\hline FLT3 & 639 & 308 & 4 & 2 & 0.01053 & 0.33 & 0.26 & 0.04 & 0.06 & 0.04 & 0.04 \\
\hline DHX8 & 142 & 89 & 2 & 2 & 0.00007 & 0.25 & 0.23 & 0.06 & 0.09 & 0.04 & 0.05 \\
\hline$F G R$ & 92 & 78 & 2 & 1 & 0.00006 & 0.33 & 0.12 & 0.16 & 0.18 & 0.11 & 0.16 \\
\hline TXNIP & 9 & 6 & 1 & 1 & $<1.0 \mathrm{E}-5$ & 0.09 & 0.16 & 0.25 & 0.33 & 0.21 & 0.29 \\
\hline GLUL & 44 & 24 & 2 & 2 & 0.00012 & 0.07 & 0.12 & 0.28 & 0.12 & 0.24 & 0.28 \\
\hline PDCDGIP & 359 & 215 & 4 & 2 & 0.00058 & 0.04 & 0.06 & 0.29 & 0.15 & 0.33 & 0.30 \\
\hline$D G K E$ & 136 & 71 & 1 & 0 & 0.00005 & 0.02 & 0.05 & 0.23 & 0.05 & 0.28 & 0.25 \\
\hline PTTGIIP & 174 & 116 & 2 & 2 & 0.00005 & 0.04 & 0.09 & 0.15 & 0.20 & 0.13 & 0.15 \\
\hline EPHA2 & 133 & 93 & 1 & 1 & 0.00021 & 0.03 & 0.17 & 0.11 & 0.03 & 0.20 & 0.10 \\
\hline
\end{tabular}


Table 3 Causal genes with empirical power over 0.2 using any weighting scheme (Continued)

\begin{tabular}{|c|c|c|c|c|c|c|c|c|c|c|c|}
\hline \multicolumn{12}{|l|}{ SBP } \\
\hline MAP4 & 542 & 392 & 7 & 5 & 0.05824 & 0.27 & 0.27 & 0.14 & 0.17 & 0.09 & 0.14 \\
\hline FLT3 & 639 & 308 & 4 & 2 & 0.00841 & 0.20 & 0.18 & 0.04 & 0.04 & 0.03 & 0.03 \\
\hline DHX8 & 142 & 89 & 2 & 2 & 0.00002 & 0.25 & 0.25 & 0.02 & 0.10 & 0.03 & 0.03 \\
\hline S100A6 & 14 & 11 & 2 & 2 & 0.00003 & 0.27 & 0.09 & 0.06 & 0.05 & 0.05 & 0.05 \\
\hline KRT23 & 79 & 31 & 2 & 1 & 0.00016 & 0.24 & 0.24 & 0.04 & 0.03 & 0.02 & 0.02 \\
\hline TXNIP & 9 & 6 & 1 & 1 & $<1.0 \mathrm{E}-5$ & 0.09 & 0.14 & 0.20 & 0.26 & 0.14 & 0.22 \\
\hline GLUL & 44 & 24 & 2 & 2 & 0.00006 & 0.04 & 0.09 & 0.27 & 0.11 & 0.22 & 0.28 \\
\hline PDCD6IP & 359 & 215 & 4 & 2 & 0.00027 & 0.04 & 0.08 & 0.33 & 0.17 & 0.36 & 0.33 \\
\hline SCAP & 114 & 83 & 1 & 1 & 0.00003 & 0.06 & 0.17 & 0.40 & 0.39 & 0.24 & 0.36 \\
\hline EPHA2 & 133 & 93 & 1 & 1 & 0.00018 & 0.04 & 0.27 & 0.21 & 0.10 & 0.34 & 0.22 \\
\hline
\end{tabular}

${ }^{1}$ Number of causal single-nucleotide polymorphisms (SNPs) is computed based on the used annotation file (not based on the answers).

${ }^{2}$ Total \% variance explained is computed by adding the percent variance explained by each SNP based on the used annotation file (not based on the answers). Bolded text indicates the empirical power greater than 0.2 .

$D B P$, diastolic blood pressure; $M-B$, Madsen-Browning; $S B P$, systolic blood pressure.

more desirable. Indeed we found that the SKAT default weighting scheme (which heavily downweights common variants) performed better for such genes. We also found that this SKAT default weighting scheme behaved similarly to other weighting schemes after eliminating all common SNPs. In contrast, we observed that an equal weighting scheme performed the best for $M A P 4$ and FLT3, both of which included a common variant with a large effect.

However, we found that SKAT with all 3 weighting schemes performed poorly. Overall power across all causal genes was about 0.05 , which was almost identical to the type I error rate. We suspect that this poor performance is partly due to a small sample size. Wu et al [6] previously demonstrated that SKAT, as with other rare variant methods, is severely hindered by small sample sizes, and this data set was no exception. Lin and Tang [9] had previously compared several rare variant methods and found that SKAT is very conservative when sample size and the level $\alpha$ for calculating power are small. Our results were also consistent with a study performed by Daye et al [10] that showed at a sample size of 200, SKAT performs at or around the $\alpha=0.05$ level regardless of coverage.

Many GAW18 investigators that analyzed only unrelated individuals (with a sample size of 142) have also observed poor performance. Because of high sequencing costs, small sample sizes will continue to be a problem with rare variant analysis. We observed that analysis using all related individuals (with a sample size of 847) performed much better [11]. For example, results based on a burden test using all related individuals provided an empirical power of 0.99 for MAP4. SKAT was recently extended to handle related individuals for continuous phenotypes [12]. We expect that if the extended SKAT were applied to all related individuals in GAW18 data, it would provide better performance for detecting rare variants.
To apply both burden and nonburden tests for a gene, it is necessary to know which SNPs are contained in the gene. GAW18 performed WGS of 1043 individuals of Mexican American heritage with an average $60 x$ sequencing depth, with a goal of finding novel SNPs. However, this creates a problem for applying these rare variant approaches because available annotation information does not contain these novel variants. In particular, among 1458 causal SNPs in the Answers, only 731 SNPs were contained in the annotation file that was constructed based on the 1000 Genomes Project. In addition to a small sample size, we suspect that our results were also affected by our use of incomplete annotation information. Although the results and issues presented in this article were based on GAW18 data, they may be shared with other sequencing studies.

\section{Competing interests}

The authors declare that they have no competing interests.

\section{Authors' contributions}

CM performed the statistical analyses and drafted the manuscript. YJS designed the overall study and drafted the manuscript. Both authors read and approved the final manuscript.

\section{Acknowledgements}

The work was sponsored by National Institutes of Health $(\mathrm{NIH})$ grants HL086694, HL107552, and HL111249. The GAW18 WGS data were provided by the T2D-GENES (Type 2 Diabetes Genetic Exploration by Next-generation sequencing in Ethnic Samples) Consortium, which is supported by $\mathrm{NIH}$ grants U01 DK085524, U01 DK085584, U01 DK085501, U01 DK085526, and U01 DK085545. The other genetic and phenotypic data for GAW18 were provided by the San Antonio Family Heart Study and San Antonio Family Diabetes/Gallbladder Study, which are supported by NIH grants P01 HL045222, R01 DK047482, and R01 DK053889. The GAW is supported by NIH grant R01 GM031575.

This article has been published as part of BMC Proceedings Volume 8 Supplement 1, 2014: Genetic Analysis Workshop 18. The full contents of the supplement are available online at http://www.biomedcentral.com/bmcproc/ supplements/8/S1. Publication charges for this supplement were funded by the Texas Biomedical Research Institute. 


\section{References}

1. Manolio TA, Collins FS, Cox NJ, Goldstein DB, Hindorff LA, Hunter DJ, McCarthy MI, Ramos EM, Cardon LR, Chakravarti A, et al: Finding the missing heritability of complex diseases. Nature 2009, 461:747-753.

2. Bansal V, Libiger O, Torkamani A, Schork NJ: Statistical analysis strategies for association studies involving rare variants. Nat Genet 2010, 11:773-785.

3. Cohen $\mathrm{JC}$, Boerwinkle $\mathrm{E}$, Mosley TH, Hobbs $\mathrm{HH}$ : Sequence variations in PCSK9, low LDL, and protection against coronary heart disease. $N$ Engl J Med 2006, 354:1264-1272.

4. Ionita-Laza I, Buxbaum JD, Laird NM, Lange C: A new testing strategy to identify rare variants with either risk or protective effect on disease. PLoS Genet 2011, 7:e1001289

5. Neale BM, Rivas MA, Voight BF, Altshuler D, Devlin B, et al: Testing for an unusual distribution of rare variants. PLoS Genet 2011, 7:e1001322.

6. Wu MC, Lee $S, C a i T$, Li Y, Boehnke M, Lin X: Rare variant association testing for sequencing data using the sequence kernel association test (SKAT). Am J Hum Genet 2011, 89:82-93.

7. Madsen $B E$, Browning SR: A groupwise association test for rare mutations using a weighted sum statistic. PLoS Genet 2009, 5:e1000384.

8. Purcell $S$, Neale B, Todd-Brown K, Thomas L, Ferreira MAR, Bender D, Maller J, Sklar P, de Bakker PIW, Daly MJ, et al: PLINK: a toolset for wholegenome association and population-based linkage analysis. Am J Hum Genet 2007, 81:559-575.

9. Lin DY, Tang ZZ: A general framework for detecting disease associations with rare variants in sequencing studies. Am J Hum Genet 2011, 89:354-367.

10. Daye ZJ, Li H, Wei Z: A powerful test for multiple rare variants association studies that incorporates sequencing qualities. Nucleic Acids Res 2012, 40:e60

11. Sung YJ, Basson J, Rao DC: Whole genome sequence analysis of the simulated systolic blood pressure in Genetic Analysis Workshop 18 family data: long-term average and collapsing methods. BMC Proc 2014 8(Suppl 1):S12.

12. Chen $H$, Meigs JB, Dupuis J: Sequence kernel association test for quantitative traits in family samples. Genet Epidemiol 2013, 37:196-204.

doi:10.1186/1753-6561-8-S1-S10

Cite this article as: Mallaney and Sung: Rare variant analysis of blood pressure phenotypes in the Genetic Analysis Workshop 18 whole genome sequencing data using sequence kernel association test. $B M C$ Proceedings 2014 8(Suppl 1):S10.

\section{Submit your next manuscript to BioMed Central and take full advantage of:}

- Convenient online submission

- Thorough peer review

- No space constraints or color figure charges

- Immediate publication on acceptance

- Inclusion in PubMed, CAS, Scopus and Google Scholar

- Research which is freely available for redistribution

Submit your manuscript at www.biomedcentral.com/submit
Ciomed Central 